\title{
Algorithms and Data Structures for Truncated Hierarchical B-splines
}

\author{
Gábor Kiss ${ }^{1}$, Carlotta Giannelli² ${ }^{2}$, and Bert Jüttler ${ }^{2}$ \\ 1 Doctoral Program "Computational Mathematics" \\ 2 Institute of Applied Geometry \\ Johannes Kepler University Linz, Altenberger Str. 69, 4040 Linz, Austria \\ e-mail: gabor.kiss@dk-compmath.jku.at, carlotta.giannelli@jku.at, \\ bert.juettler@jku.at
}

\begin{abstract}
Tensor-product B-spline surfaces are commonly used as standard modeling tool in Computer Aided Geometric Design and for numerical simulation in Isogeometric Analysis. However, when considering tensor-product grids, there is no possibility of a localized mesh refinement without propagation of the refinement outside the region of interest. The recently introduced truncated hierarchical B-splines (THBsplines) [5] provide the possibility of a local and adaptive refinement procedure, while simultaneously preserving the partition of unity property. We present an effective implementation of the fundamental algorithms needed for the manipulation of THB-spline representations based on standard data structures. By combining a quadtree data structure which is used to represent the nested sequence of subdomains - with a suitable data structure for sparse matrices, we obtain an efficient technique for the construction and evaluation of THB-splines.
\end{abstract}

Keywords: hierarchical tensor-product B-splines; truncated basis; THB-splines; isogeometric analysis; local refinement

\section{Introduction}

The de facto standard in computer aided geometric design is the tensor-product B-spline model together with its non-uniform rational extension (NURBS). Among other fundamental properties, like minimum support, efficient refinement and degree-elevation algorithms, B-splines are nonnegative and form a partition of unity. This implies that a B-spline curve/surface is completely contained in the convex hull of a certain set of points, usually referred to as control net. The shape of the control net directly influences the shape of the B-spline representation, so that the designer can use it to manipulate the corresponding parametric representation in a fairly intuitive way. Unfortunately, an unavoidable drawback of the tensor-product structure is a global nature of the mesh refinement which excludes the possibility of a local refinement scheme as illustrated in Figure 1( $\mathrm{a}-\mathrm{c})$. 


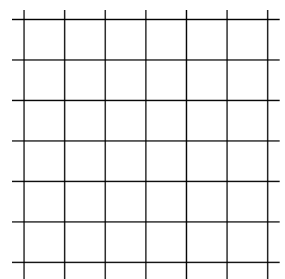

(a) initial grid

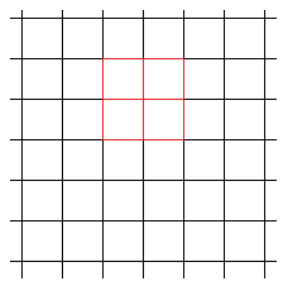

(b) area of interest

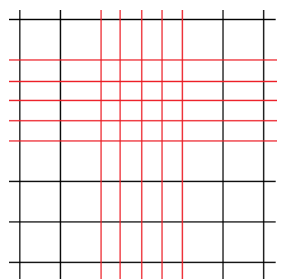

(c) knot insertion

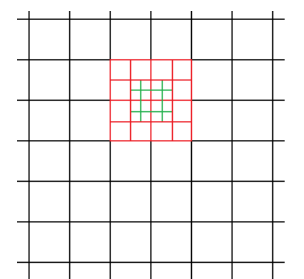

(d) hierarchical grid

Fig. 1. Adaptive refinement of an initial tensor-product grid (a) with respect to a localized region (b) may be achieved by avoiding a propagation of the refinement due to the tensor-product structure (c) through a hierarchical approach (d).

Despite an increasing interest in the identification of adaptive spline spaces and related applications, see e.g., $[7,15,18]$, local mesh refinement remains a non-trivial and computationally expensive issue. A suitable trade-off between the quality of the geometric representation (in terms of degrees of freedom needed to obtain a certain accuracy) and the complexity of the mesh refinement algorithm has necessarily to be taken into account. Different approaches have been proposed which all extend the standard tensor-product model by allowing $\mathrm{T}$ junctions between axis aligned mesh segments. Among others, this led to the introduction of hierarchical B-splines (HB-splines) [4, 11, 12], T-splines [16], polynomial splines over $\mathrm{T}$-meshes [2] and - more recently - truncated hierarchical B-splines (THB-splines) [5] and locally refined B-splines [3].

The idea of performing surface modeling by manipulating the parametric representation at different levels of details was originally proposed by Forsey and Bartels [4]. In order to localize the editing of detailed features, the refinement is iteratively adapted on restricted patches of the surface in terms of a sequence of overlays with nested knot vectors. Subsequently, Kraft $[11,12]$ showed that the hierarchical structure enforced on the mesh refinement procedure can be complemented by a simple and automatic identification of basis functions which naturally generalizes some of the fundamental properties of tensor-product Bsplines - such as nonnegativity and linear independence - to the case of HBsplines.

The multilevel approach allows to break the rigidity of a tensor-product configuration by simultaneously preserving a highly organized structure as shown in Figure 1(d). An example of hierarchical refinements over rectangular-shape regions is presented in Figure 2.

The hierarchical B-spline model found applications in data interpolation and approximation $[10,11,13]$, as well as in finite element and isogeometric analysis $[1,14,18]$. Alternative spline hierarchies were also considered in the literature, see e.g., $[9,19]$.

Kraft's basis for HB-splines does not possess the partition of unity property without additional scaling and it possesses only limited stability properties. 
Truncated hierarchical B-splines [5] have the potential to overcome these limitations and provide improved sparsity properties. They were introduced as a possible extension of normalized tensor-product B-splines to suitably handle the local refinement in adaptive surface approximation algorithms. This multilevel scheme was also generalized and further investigated in [6], where particular attention was devoted to the stability analysis of the proposed hierarchical construction.

In virtue of the multilevel nature of the hierarchical B-spline approach, the natural choice in terms of data structures is a tree-like representation where a given refinement level correspond to a certain level of depth in the tree [4]. Related and alternative solutions were also further investigated. An algorithm for scattered data interpolation and approximation by multilevel bicubic B-splines based on a hierarchy of control lattices was described in [13]. An implementation of hierarchical B-splines in terms of a tree data structure whose nodes represent the B-splines from different levels was recently presented in [1]. Another solution consists of storing in each node of the tree the data related to a knot span of a certain level, in particular the significant basis functions acting on it [14].

The goal of the present paper is to introduce an effective implementation of data structures and algorithms for the newly introduced THB-splines. To represent the subdomain hierarchy we use a quadtree data structure in combination with sparse matrices. The quadtree provides an efficient and dynamic data structure for representing the subdomains. It also facilitates the needed update which may be caused by an iterative refinement process. One key motivation for this choice is to reduce the memory overhead in need for storing the subdomain hierarchy as much as possible. The selection of (possibly truncated) basis functions proceeds as described in [5] by means of certain queries which use the quadtree. The result is encoded by a sequence of sparse matrices. The quadtree and the related sparse matrices are initially created and subsequently updated during the refinement procedure. For the hierarchical spline evaluation algorithm, however, only the access to the sparse matrices is required. This leads to a reasonable trade-off with respect to memory and time consumption during both the construction of THB-splines from an underlying subdomain hierarchy and their evaluation for given parameter values.

The paper is organized as follows. In Section 2 we describe the hierarchical approach to adaptive mesh refinement together with the definition and evaluation of the THB-spline basis. Section 3 introduces the data structures and algorithms used for the representation of the subdomain hierarchy, while Section 4 explains the construction of the matrices needed during the THB-spline evaluation in more detail. Some numerical results are then presented in Section 5 to illustrate the performance of our approach, while the extension of the proposed approach to more general knot configurations and refinements is discussed in Section 6. Finally, Section 7 concludes the paper. 

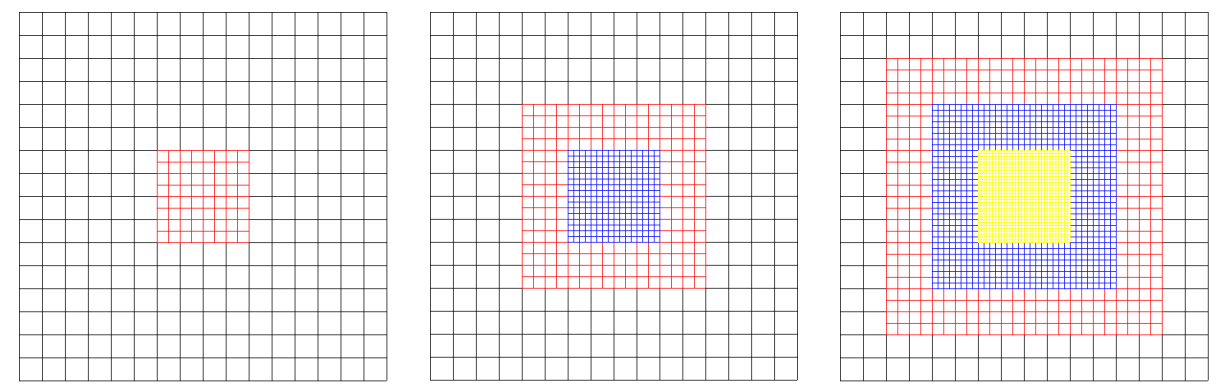

Fig. 2. An example of hierarchical refinement over rectangular-shape regions where the central area of the mesh is always refined up to the maximum level of detail: two levels (left), three levels (middle) and four levels (right).

\section{THB-splines}

We define an adaptive extension of the classical tensor-product B-spline construction in terms of a certain number $N$ of hierarchical levels which correspond to an increasing level of detail. At each refinement step we select a specific tensor-product grid associated with the current level. Provided that the sequence of tensor-product grids corresponds to a nested sequence of spline spaces $V^{0}, \ldots, V^{N-1}$ which satisfies

$$
V^{\ell-1} \subset V^{\ell}
$$

for $\ell=1, \ldots, N-1$, the hierarchical framework allows to consider different types of grid refinement. Following the notation of [17], the superscript $\ell$ denotes the hierarchical level $\ell$ throughout the paper.

The present paper focuses on the bivariate tensor-product case. However, the framework can easily be adapted to the multivariate setting and even to more general spline spaces $[5,6]$. Nevertheless, even if the representation model we are going to introduce may in principle be used to handle non-uniform mesh refinement and even spaces generated by degree elevation, we will consider only the dyadic uniform case throughout this paper. The modifications which are required to extend the proposed approach to more general knot configurations are discussed in Section 6 .

More precisely, we assume that the coarsest spline space $V^{0}$ is spanned by bivariate tensor-product $\mathrm{B}$-splines with respect to two bi-infinite uniform knot sequences. The finer spaces $V^{\ell}$ are obtained by iteratively applying dyadic subdivision, i.e., each cell of the original tensor-product grid is split uniformly into four cells.

Let $\Omega^{0}$ be a rectangular planar domain whose edges are aligned with the tensor-product grid of $V^{0}$, and let $\left\{\Omega^{\ell}\right\}_{\ell=0, \ldots, N-1}$ be a nested sequence of subdomains so that

$$
\Omega^{\ell-1} \supseteq \Omega^{\ell}
$$




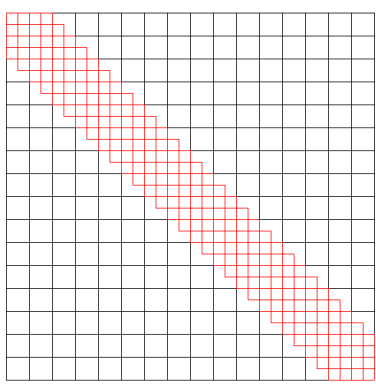

(a) $\Omega^{0} \supseteq \Omega^{1}$

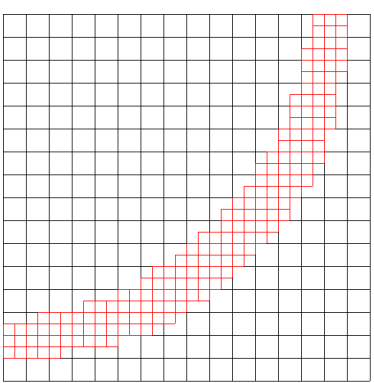

(d) $\Omega^{0} \supseteq \Omega^{1}$

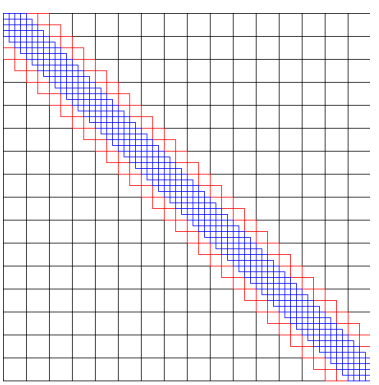

(b) $\Omega^{0} \supseteq \Omega^{1} \supseteq \Omega^{2}$

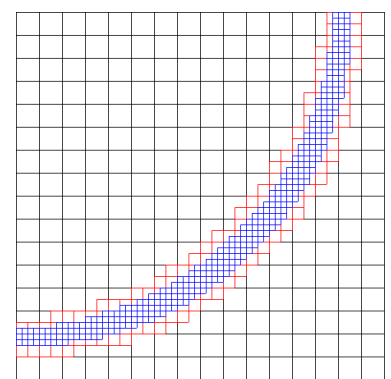

(e) $\Omega^{0} \supseteq \Omega^{1} \supseteq \Omega^{2}$

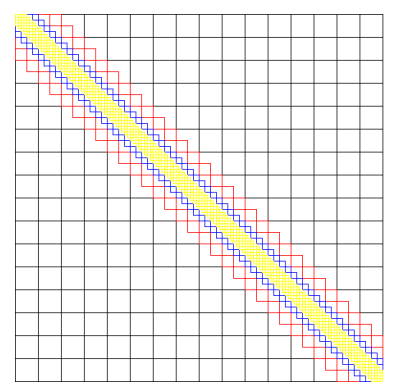

(c) $\Omega^{0} \supseteq \Omega^{1} \supseteq \Omega^{2} \supseteq \Omega^{3}$

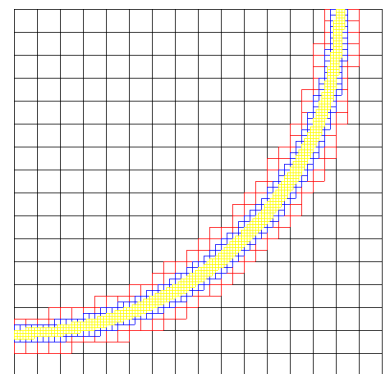

(f) $\Omega^{0} \supseteq \Omega^{1} \supseteq \Omega^{2} \supseteq \Omega^{3}$

Fig. 3. Two nested sequences of subdomains - indicated as linear (top) and curvilinear (bottom) in Example 1. They satisfy relation (1) with respect to two (left), three (middle) and four (right) hierarchical levels.

for $\ell=1, \ldots, N-1$. Each $\Omega^{\ell}$ is defined as a collection of cells with respect to the tensor-product grid of level $\ell-1$.

Example 1. Figures 2 and 3 show three subdomain hierarchies which will be used to demonstrate the performance of our algorithms and data structures:

- rectangular (refinement over rectangular-shaped regions);

- linear (refinement along a diagonal layer);

- curvilinear (refinement along a curvilinear trajectory).

By starting with an initial tensor-product configuration at level 0 , the tensorproduct grid associated with level $\ell+1$ is obtained by subdividing any cell of the previous level into four parts. Each subdomain $\Omega^{\ell}$ is then defined as a certain collection of cells with respect to the grid of level $\ell$ so that (1) is satisfied. Figure 2 illustrates an example of hierarchical refinement over rectangular-shape regions where the central area of the mesh is always refined up to the maximum level of detail. The other two subdomain hierarchies mentioned above are shown in Figure 3 up to four refinement levels so that $\Omega^{0} \supseteq \ldots \supseteq \Omega^{3}$.

For each hierarchical level $\ell$, with $\ell=0, \ldots, N-1$, let $\mathcal{B}^{\ell}$ be the normalized B-spline basis of the spline space $V^{\ell}$ with respect to a certain degree $(d, d)$ 
defined on corresponding nested knot sequences. We say that

$$
\beta \in \mathcal{B}^{\ell} \text { is active } \Leftrightarrow \operatorname{supp}^{0} \beta \subseteq \Omega^{\ell} \wedge \operatorname{supp}^{0} \beta \nsubseteq \Omega^{\ell+1},
$$

where $\operatorname{supp}^{0} \beta=\operatorname{supp} \beta \cap \Omega^{0}$ is a slightly modified support definition which makes local refinements possible also along the boundaries of $\Omega^{0}$. A B-spline $\beta \in \mathcal{B}^{\ell}$ is then active if it is completely contained in $\Omega^{\ell}$ but not in $\Omega^{\ell+1}$, and passive otherwise.

We may assume the initial domain $\Omega^{0}$ to be an axis-aligned box ${ }^{3}$. By denoting with $k$ the number of knot spans of level 0 along the edges of $\Omega^{0}$, which is assumed to be the same for both directions, we define a characteristic matrix $X^{\ell}$ of size $s^{\ell} \times s^{\ell}$, with $s^{\ell}=\left(2^{\ell} k+d\right)$, for $\ell=0, \ldots, N-1$. These matrices collect the information about active/passive B-splines level by level, namely

$$
X_{i, j}^{\ell}= \begin{cases}1 & \text { if } \beta_{i, j}^{\ell} \text { is active } \\ 0 & \text { otherwise }\end{cases}
$$

where $\beta_{i, j}^{\ell}$ is a $\mathrm{B}$-spline of level $\ell$. The indices $i, j$ are chosen such that exactly the B-splines $\beta_{i, j}^{\ell}$ with $i, j=1, \ldots, s^{\ell}$ are non-zero on $\Omega^{0}$.

Definition 1 ([11, 12], extended in [18]). The hierarchical B-spline $(H B-$ spline) basis $\mathcal{H}$ is defined as the set of all active $B$-splines defined over the tensor-product grid of each level,

$$
\mathcal{H}=\bigcup_{\ell=0, \ldots, N-1}\left\{\beta_{i, j}^{\ell} \in \mathcal{B}^{\ell}: X_{i, j}^{\ell}=1\right\}
$$

A spline function represented in the hierarchical B-spline basis is then defined as a linear combination of active B-splines from different levels in the hierarchy. In order to evaluate the considered spline in a given point of the domain, the contribution of all the active B-splines (from the minimum to the maximum level of basis functions whose support is non-zero on that point) has to be computed and then added together. The cost of the hierarchical evaluation algorithm is then quadratic and linear with respect to the degree (B-spline evaluation) and the number of levels, respectively.

Truncated hierarchical B-splines $[5,6]$ form a different basis for the same multilevel B-spline space. The key idea behind this alternative hierarchical construction is to properly exploit the refinable nature of the $\mathrm{B}$-spline basis which allows to express a B-spline of level $\ell$ in terms of $(d+2)^{2}$ functions which belong to level $\ell+1$ and of certain binomial coefficients scaled by a factor $2^{-d}$ with respect to any dimension. By using this subdivision rule, any function $\tau \in V^{\ell}$ can be represented according to a two-scale relation with respect to the basis $\mathcal{B}^{\ell+1}$ of $V^{\ell+1}$, namely

$$
\tau=\sum_{\beta \in \mathcal{B}^{\ell+1}} c_{\beta}^{\ell+1}(\tau) \beta,
$$

\footnotetext{
${ }^{3}$ Different shapes are easily identified at subsequent levels as shown in Figure 3.
} 
with certain coefficients $c_{\beta}^{\ell+1}(\tau) \in \mathbb{R}$. The truncation of $\tau \in V^{\ell}$ with respect to $\mathcal{B}^{\ell+1}$ and $\Omega^{\ell+1}$ is the function trunc ${ }^{\ell+1} \tau \in V^{\ell+1}$ defined as:

$$
\operatorname{trunc}^{\ell+1} \tau=\sum_{\beta \in \mathcal{B}^{\ell+1}, \operatorname{supp} \beta \nsubseteq \Omega^{\ell+1}} c_{\beta}^{\ell+1}(\tau) \beta .
$$

The overall truncation of a hierarchical B-spline $\beta \in \mathcal{B}^{\ell} \cap \mathcal{H}$ is defined by recursively applying the truncation with respect to the different levels,

$$
\operatorname{trunc} \beta=\operatorname{trunc}^{N-1}\left(\operatorname{trunc}^{N-2} \ldots\left(\operatorname{trunc}^{\ell+1} \beta\right)\right) .
$$

By recursively discarding the contribution of active B-splines of subsequent levels from coarser B-splines, we obtain the definition of the truncated basis.

Definition 2 ([5]). The truncated hierarchical B-spline (THB-spline) basis $\mathcal{T}$ is defined by

$$
\mathcal{T}=\left\{\operatorname{trunc} \beta_{i, j}^{\ell}: X_{i, j}^{\ell}=1, \ell=0, \ldots, N-2\right\} \cup\left\{\beta_{i, j}^{N-1}: X_{i, j}^{N-1}=1\right\} .
$$

Truncated hierarchical B-spline are linearly independent, non-negative, form a partition of unity and preserve the nested nature of the spline spaces [5]. Moreover, the construction of THB-splines is strongly stable with respect to the supremum norm provided that the knot vectors satisfy certain reasonable assumptions — see [6] for more details.

In addition to the characteristic matrices $\left\{X^{\ell}\right\}_{\ell=0}^{N-1}$, we consider another sequence of matrices $\left\{C^{\ell}\right\}_{\ell=0}^{N-1}$ of the same size and with the same sparsity pattern, i.e. $X_{i, j}^{\ell}=0$ implies $c_{i, j}^{\ell}=0$. These matrices store the coefficients associated to the (active) basis functions in the representation of a spline function with respect to the truncated basis. The following simple algorithm performs the evaluation of a hierarchical spline function which is represented in terms of THB-splines.

Algorithm EVAL_THB (seqmat X, seqmat C, int D, int LMAX, float U,V)

$\backslash \backslash$ seqmat $X$ is the sequence of characteristic matrices, i.e., $X$ [L] is the characteristic matrix of level $\mathrm{L}$

$\backslash \backslash$ seqmat $C$ is the sequence of coefficient matrices associated to the spline function $f$, i.e., $\mathrm{C}[\mathrm{L}]$ is the coefficient matrix of level $\mathrm{L}$

$\backslash \backslash$ int $\mathrm{D}$ is the degree in both directions

$\backslash \backslash$ int LMAX is the maximum refinement level $N-1$

$\backslash$ float $U, V$ are evaluation parameters

Identify the $(D+1) \times(D+1)$ sub-matrix $M$ of $C[0]$ which contains the coefficients of those $\mathrm{B}$-splines of level 0 that are non-zero at $(\mathrm{U}, \mathrm{V})$

for $L=1$ to LMAX do \{

Generate the matrix S by applying one step of $\mathrm{B}$-spline subdivision to $\mathrm{M}$ Identify the $(D+1) \times(D+1)$ sub-matrix $T$ of $S$ which contains the coefficients of those B-splines of level L that are non-zero at $(\mathrm{U}, \mathrm{V})$

for each pair of indices $i, j$ in $T$ do \{

if $X[L](i, j)==1$ then $T(i, j)=C[L](i, j)\}$ 
$\mathrm{M}=\mathrm{T}\}$

return the value $\mathrm{f}$ obtained by applying de Boor's algorithm to $\mathrm{M}$

In this algorithm, the sub-matrices $\mathrm{M}, \mathrm{S}$, and $\mathrm{T}$ at a certain level are always accessed by global indices, i.e., indices with respect to the entire array of all tensor-product splines of that level. The following proposition clarifies the connection between the evaluation algorithm and the truncated hierarchical B-spline basis.

Theorem 1. The value $f(u, v)$ computed by the algorithm is the value of a function represented in the THB-spline basis.

This can be proved by applying the algorithm to Kronecker-type coefficient data, where exactly one coefficient is nonzero and equals 1 . This corresponds to the evaluation of a truncated basis function.

The cost of the THB-spline evaluation algorithm EVAL_THB is equal to $N-1$ times the application of the B-spline subdivision rule plus the cost due to the standard de Boor's algorithm. Consequently, it grows linearly with the number of levels and quadratically with the degree of the splines. This is similar to the costs needed for evaluating the classical (non-truncated) hierarchical B-splines. The computational cost could be further reduced

- by starting the for loop at the minimum level of functions which are active at the given point $(u, v)$, and

- by stopping it at the maximum level of functions which are active at this point.

With this modification, the computational costs grows linearly with the number of levels which are active at the given point. This number can be controlled by choosing a suitable refinement strategy.

The following sections discuss data structures and algorithms for manipulating and storing the subdomain hierarchy and for representing the characteristic matrices and coefficient matrices.

\section{Representing and manipulating the subdomain hierarchy}

The domain $\Omega^{0}$ is now assumed to be a box consisting of $2^{n} \times 2^{n}$ cells of the coarsest tensor-product grid, where $n$ is a non-negative integer. This assumption is made in order to facilitate the use of a quadtree data structure. Moreover, in order to simplify the implementation, the edges of the coarsest tensor-product grid should have the length $2^{M-1}$, where $M$ is the maximum number of levels, i.e. $N \leq M$. Under this assumption, all coordinates of bounding boxes in the algorithms presented below are integers. Alternatively, one may use other exact data types than integers (e.g. rational numbers), thereby eliminating the restriction on the number of levels. 


\subsection{The subdomain hierarchy quadtree}

We represent the entire subdomain hierarchy by a single quadtree. Each node of the quadtree takes the form

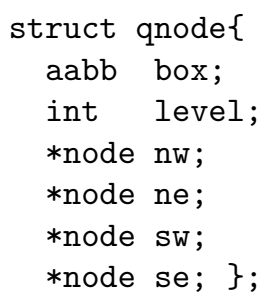

\begin{tabular}{|c|c|c|c|}
\hline \multicolumn{2}{|c|}{ box } & \multicolumn{2}{c|}{ level } \\
\hline nw & ne & sw & se \\
\hline
\end{tabular}

where the axis-aligned bounding box aabb box is characterized by coordinates of its upper left and lower right corner, level defines the highest level in which the box is completely contained and nw, ne, sw, se are pointers to the four children of the node. These children represent the northwestern, northeastern, southwestern and southeastern part of the box after the dyadic subdivision. All pointers to these children are set to null until the node is created during the insertion process, which is described by the INSERTBOX algorithm below.

Let $\Omega^{\ell}=\bigcup_{i} b_{i}^{\ell}$, where each $b_{i}^{\ell}$ is a collection of cells forming a rectangular box. During the creation of the quadtree which represents the subdomain hierarchy, for each level $\ell$, we insert all boxes $b_{i}^{\ell}$ which define $\Omega^{\ell}$. The following recursive algorithm performs the insertion of a box $b_{i}^{\ell}$ into the quadtree:

Algorithm INSERTBOX (box $B$, gnode $Q$, int $L$ )

$\backslash \backslash$ box $B$ is the box which will be inserted

$\backslash \backslash$ qnode $Q$ is the current node of the quadtree

$\backslash$ int $\mathrm{L}$ is the level

if $B==Q$.box then \{

Q. level $=\mathrm{L}$

visit all nodes in the subtree with root Q; if the level of a node is less than $\mathrm{L}$ then increase it to $\mathrm{L}\}$

else \{

for child in $\{\mathrm{Q} . \mathrm{nw}, \mathrm{Q} . \mathrm{ne}, \mathrm{Q} . \mathrm{Sw}, \mathrm{Q} . \mathrm{se}\}$ do \{

if child $!=$ null then \{

If $B \cap Q \cdot b o x \neq \emptyset$ then INSERTBOX $(B \cap Q \cdot b o x$, child, L) $\}$ else \{

create the box childbox of child

if $\mathrm{B} \cap \mathrm{childbox} \neq \emptyset$ then

create the node child

set child.box to childbox, child.level to Q.level and the four children to null

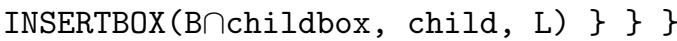

After each box insertion we perform a cleaning step, visiting all sub-trees and deleting those where all nodes have the same level. This reduces the depth of the tree to a minimal value and optimizes the performance of all algorithms. 

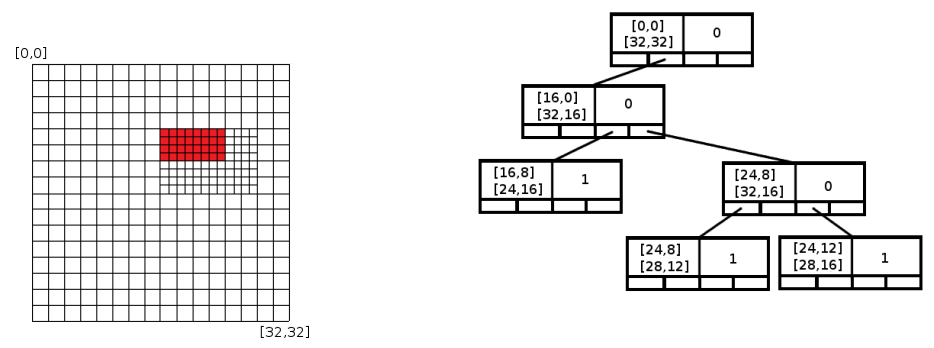

Fig. 4. Initial subdomain structure (left) and corresponding quadtree (right) which stores the boxes related to level 0 and 1 in the hierarchy. The box $b=[16,8] \times[24,12]$ (red) has to be inserted into the quadtree at level 2 .

Example 2. To explain the INSERTBOX algorithm, we consider the subdomain hierarchy composed of three levels $(N=3)$, two of which (level 0 and 1$)$ are initially present. This is shown in Figure 4, together with the related quadtree representation. The domain $\Omega^{0}$ has $k=16$ edges of length $2^{N-2}=2$. The box $b=[16,8] \times[24,12]$ will be inserted at level 2 into the hierarchy. The cells with respect to the tensor-product grid of level 1 covered by $b$ are depicted in red in Figure 4.

The execution of the algorithm is illustrated in Figure 5. At each step, we highlight the current node $\mathrm{Q}$ and the corresponding box in the subdomain hierarchy (Figure 5, right and left column, respectively). The insertion starts by considering the root of the tree, where the box $b$ is compared with the axisaligned bounding box stored in the root. Since these two boxes are not the same, the level of the root remains unchanged.

Subsequently, we have to identify which boxes between the ones stored in the four children of the root overlap with $b$, see Figure $5(\mathrm{a})$. In this case $b$ is completely contained in the box represented by the ne child of the root. The recursive call of INSERTBOX is therefore applied to this child only. The situation in Figure 5(b) is similar to the previous case. After the split, the algorithm is recursively applied to the sw child.

In the third step shown in Figure 5(c) instead, the box $b$ overlaps with the boxes related to two children (nw and ne) of the current node. Then, $b$ is also subdivided and the recursion is called on both children.

Figure 5(d) shows the last step executed by the insertion of the box $b$. Two new nodes are created and inserted into the quadtree. Since these nodes coincide with the two parts of $b$, we set their level to 2. Clearly, the box to be inserted does not necessarily become a single node of the quadtree but it may be stored into several nodes. 

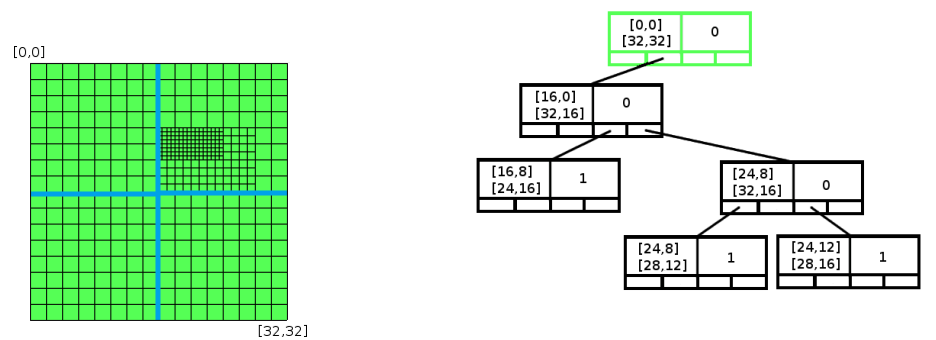

(a) first split (left) and quadtree (right)
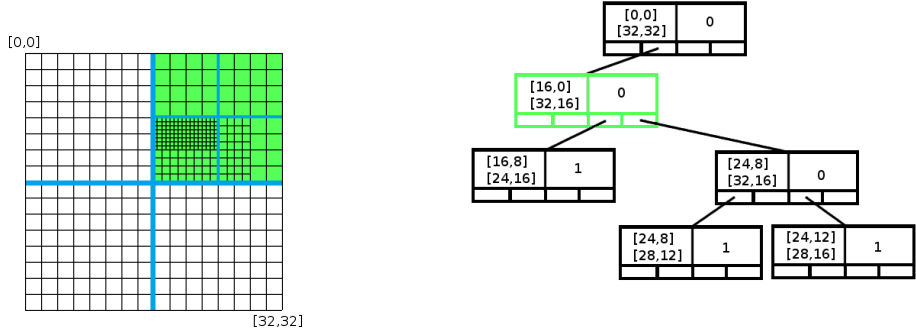

(b) second split (left) and quadtree (right)
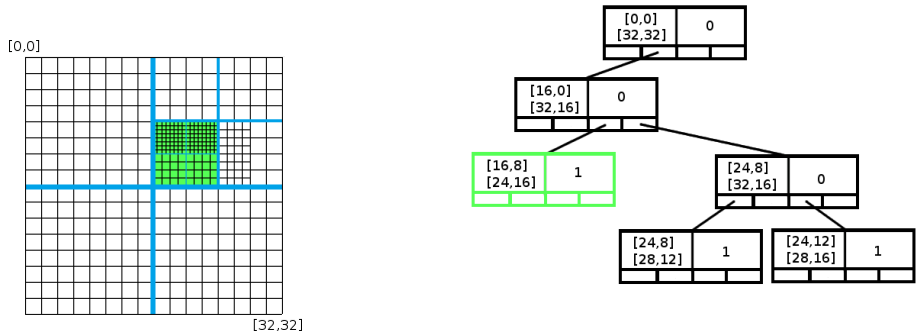

(c) third split (left) and quadtree (right)

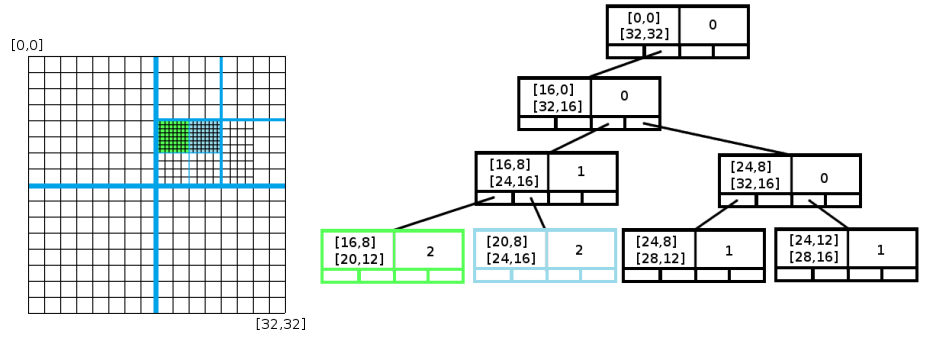

(d) two new boxes (left) are inserted into the quadtree (right)

Fig. 5. Different steps performed by the INSERTBOX function to insert the box $b=$ $[16,8] \times[24,12]$ into the subdomain hierarchy of Figure 4 . The subsequent splits are shown on the subdomain hierarchy (blue lines on the left) with respect to the visit of the quadtree (right). 


\subsection{Queries}

In order to create the characteristic matrices introduced in Section 2, we define three query functions on the quadtree. These queries allow to understand if all basis functions $\beta$ of a certain hierarchical level whose support is contained in a given box $b$ are active or passive.

Given a box $b$ defined as a collection of cells with respect to the tensorproduct grid of level $\ell$, the first query (QY1), returns true if

$$
b \subseteq \Omega^{\ell} \wedge \quad b \cap \Omega^{i}=\emptyset, \quad i>\ell .
$$

Thus, if QY1 returns true, then all the basis functions of level $\ell$ whose support is completely contained in the box $b$ are active, i.e., they are present in the hierarchical spline basis.

If the second query QY2 returns true then all the basis functions of level $\ell$ whose support is contained in the box $b$ are passive, i.e., they are not present in the hierarchical spline basis. This is characterized by the following condition:

$$
b \cap \Omega^{\ell}=\emptyset \quad \vee \quad b \subseteq \Omega^{\ell}, \quad \text { for some } i>\ell .
$$

The third query QY3 returns the highest level $\ell$ with the property that $\Omega^{\ell}$ contains the box $b$.

All the three queries can easily be implemented with the help of the quadtree structure described before. In particular, the structure of queries QY1 and QY2 is similar. We visit the quadtree until we find a leaf node or a node where the result of the query changes from to true to false. At that point, we can conclude the visit and return false. On the other hand, query QY3 requires a complete visit of the quadtree.

Example 3. Figure 6(b-d) shows the results of the three queries with respect to the subdomain hierarchy composed of two levels (level 0 and 1) shown on Figure 6(a) for four sampled boxes of level 0. Figures 6(b) and (c) display the results of QY1 and QY2 for $\ell=0$, respectively. The boxes in green correspond to a positive answer to the query, the red boxes to a negative one. Finally, Figure 6(d) shows the results for QY3. The green boxes correspond to answer 1 and the red ones to answer 0.

\section{Characteristic matrices}

The characteristic matrices identify the tensor-product basis functions which are present in the hierarchical basis.

\subsection{Creating characteristic matrices}

By using the quadtree structure defined in Section 3, we can generate the characteristic matrices introduced in Section 2 to represent and evaluate THB-splines. For the creation of these matrices we considered two different approaches: 


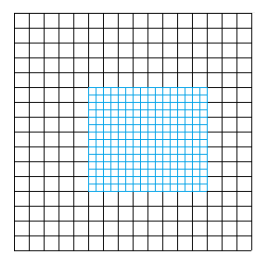

(a) level 0 and 1

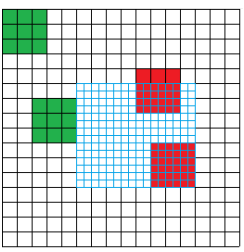

(b) QY1 for level 0

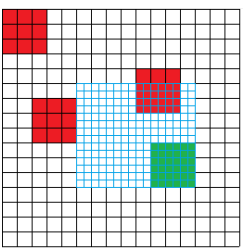

(c) QY2 for level 0

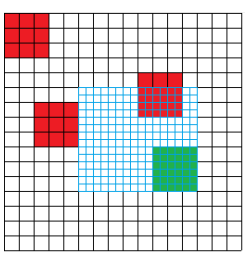

(d) results of QY3

Fig. 6. Results of the three queries functions with respect to a subdomain hierarchy (a) with two levels. In case of QY1 (b) and QY2 (c), the green/red boxes correspond to a positive/negative answer. QY3 (d) returns 1 for the green boxes and 0 for the red ones.

- the one-by-one approach where we determine the entries of the characteristic matrices one by one by applying QY3 to each single basis function;

- the all-at-once approach where we try to set as many values as possible in one single step. This requires a more sophisticated algorithm.

During the creation of the characteristic matrices by the all-at-once approach, we try to set many entries of the matrices at the same time. In order to do this, the query functions are initially called for boxes which cover the initial domain $\Omega^{0}$. The SETMAT algorithm below creates the characteristic matrices for all subdomains in the subdomain hierarchy.

\section{Algorithm SETMAT (qnode $Q$, seqmat $X$ )}

$\backslash \backslash$ qnode $Q$ is the root of the quadtree which stores the subdomain hierarchy $\backslash \backslash$ seqmat $X$ is the sequence of characteristic matrices, i.e. $X[L]$ is the characteristic matrix of level $\mathrm{L}$

for all levels L do \{

Create the index set I for all functions of level L acting on $\Omega^{0}$. I is an axis-aligned box in index space.

$\operatorname{SETBOX}(B, X[L])\}$

SETMAT calls the algorithm SETBOX. When the answer active/passive cannot be given for the current call, the considered box is split into 4 disjoint axisaligned bounding boxes and SETBOX function is recursively applied to them.

\section{Algorithm SETBOX (aabbis I, mat XL)}

$\backslash 1$ aabbis $I$ is an axis-aligned box in index space

$\backslash \backslash$ mat $\mathrm{XL}$ is a characteristic matrix of level $\mathrm{L}$

The level $\mathrm{L}$ is a global variable

Create the axis-aligned bounding box B covering all cells of level L which belong to the supports of functions with indices in I

if $\mathrm{QY1}(\mathrm{B}, \mathrm{L})$ then \{

for all indices $(i, j)$ in $I$ do XL $[i, j]=1\}$ 


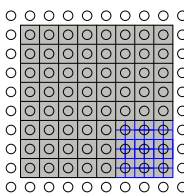

(a)

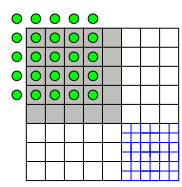

(b)

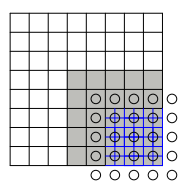

(c)

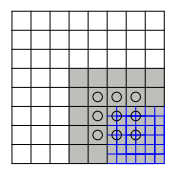

(d)

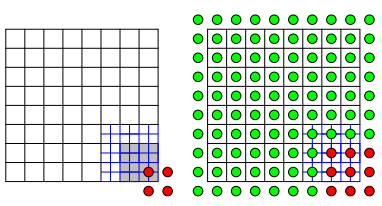

(e) (f)

Fig. 7. A subdomain hierarchy with two levels and some of the boxes I in index space (shown as circles) along with the associated bounding boxes B in parameter space (grey) considered by SETBOX when creating the characteristic matrix $X^{0}$ for this subdomain hierarchy (a-e). Active (green) and passive (red) functions of level 0 (f).

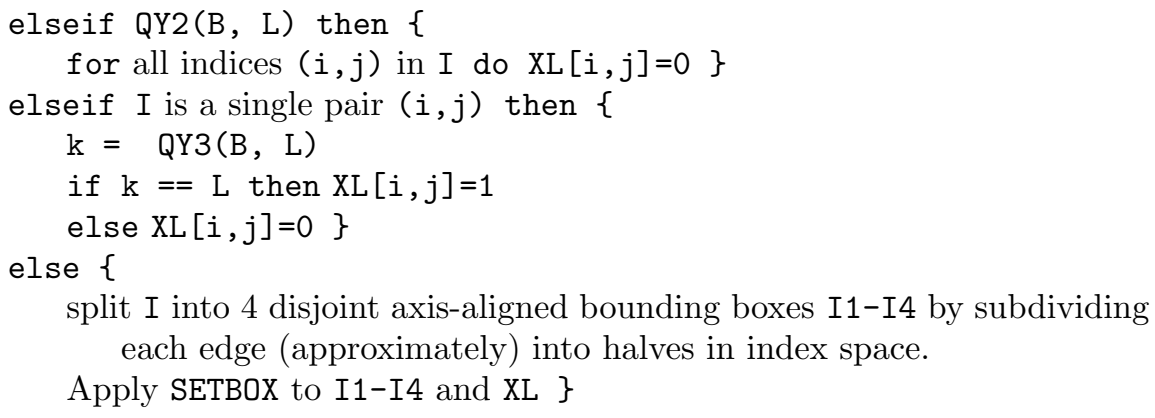

Example 4. Figure 7 shows a subdomain hierarchy with two levels, consisting of a square $\Omega^{0}$ and a subdomain $\Omega^{1}$ in the southeastern corner, which is shown in blue. The four pictures (a-e) visualize several index sets I (shown by circles) and the associated boxes B (grey) which are considered by SETBOX when creating $X^{0}$ for biquadratic splines.

Initially, SETBOX considers the entire set of basis functions (a) and concludes that it has to be subdivided. The northwestern subset is shown in (b). Query QY1 returns 1, therefore the functions are all active; no subdivision is needed. The northeastern and southwestern subsets (not shown) are dealt with similarly. The southeastern subset (c), however, has to be subdivided. Considering its northwestern subset $(\mathrm{d})$ does not lead to a conclusion again, needing another subdivision. The functions in this index set have to be analyzed one-by-one (not shown). The northeastern and southwestern subsets (not shown) are dealt with similarly. For the southeastern subset (e), however, query QY2 returns 1, therefore the functions are all passive. Finally, the procedures arrives at the correct classification of basis functions of level 0 (f).

As Example 5 shows the all-at-once approach is not necessarily faster compared to the one-by-one mentioned at the beginning of this section. However, the approach becomes considerably faster with an increasing number of levels. This is demonstrated by the next example.

Example 5. Figure 8 compares the all-at-once setting with the one-by-one method. The number of queries called by the one-by-one approach is the same 


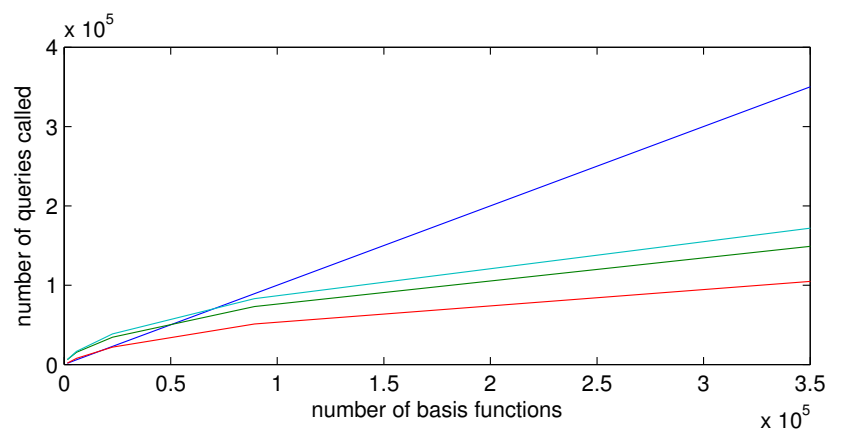

Fig. 8. The plot visualizes the number of queries needed to create the characteristic matrices for the three examples in Figure 2, 3 and 9. Compared to the all-at-once approach (cyan: linear, green: curvilinear, red: square-shaped refinement), the one-byone approach (blue: same for all examples) is faster for small numbers of levels and basis functions, but it becomes slower for higher ones.
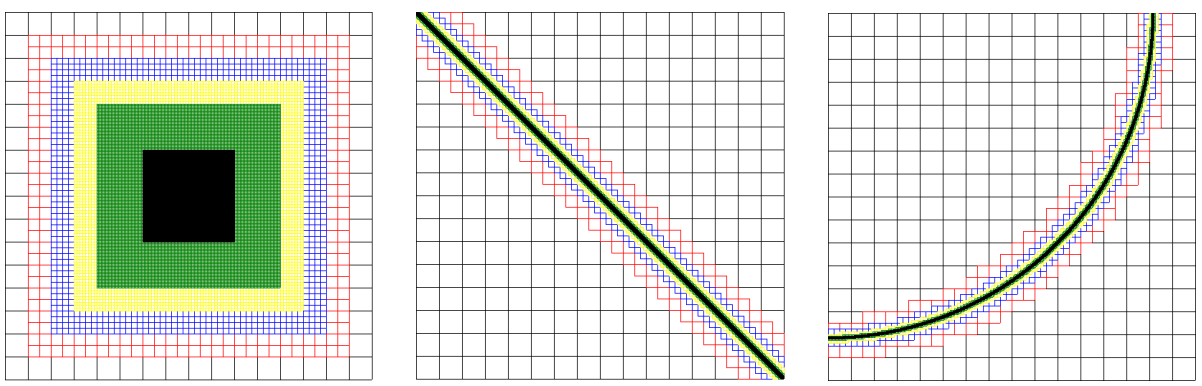

Fig. 9. The three subdomain hierarchies considered in Example 6: rectangular (left), linear (middle) and curvilinear (right) refinement, all with six levels.

for the three hierarchical refinements in Figure 9 since it depends solely on the number of basis functions. This approach is faster for small numbers of basis functions, which typically correspond to a small numbers of hierarchical levels. On the other hand, the all-at-once approach becomes faster for higher numbers of basis functions in all the three considered cases since the number of queries grows sub-linearly with respect to the number of basis functions.

\subsection{Using sparse data structures}

The representation of THB-splines in terms of characteristic matrices allows a fast look-up during the evaluation process and a simple update of the values when the underlying subdomain hierarchy changes. The drawback of this representation is the rather large memory consumption, which can exceed the 
available physical memory even for relatively small meshes and low numbers of levels. Indeed, it grows exponentially with the number of levels.

This problem can be solved by using a suitable sparse matrix data structure. For our experiments, we chose the compressed sparse column (CSC) data structure. The nonzero elements (read first by column) are stored in a onedimensional array. A second array stores the row indices corresponding to these values and a third one collects the indices into the first two arrays of the leading entry in each column [8].

As detailed in the next section, the CSC structure significantly reduces the memory consumption of our approach (see Example 6). In fact, we will observe that the memory consumption grows linearly with the number of degrees of freedom, instead of exponentially with the number of levels. In addition, the price paid for reducing the memory requirements is only a small increase of the computational time (see Examples 7 and 8).

\section{$5 \quad$ Examples}

We implemented the proposed algorithms and data structures in C++. For the manipulation of the characteristic matrices we used the sparse MATLAB representation in terms of the compressed sparse column approach mentioned at the end of the previous section. The experiments have been performed on a laptop running the Windows 7 operating system (Intel Core I5-2520 2.5GHz, 4GB RAM, 64 bit).

Example 6. We compare the memory consumption of full characteristic matrices with the memory consumption of the matrices represented in the CSC structure for the three subdomain hierarchies in Figure 9 (rectangular, linear, and curvilinear).

The experimental results in Figure 10 show that the memory needed by the sparse matrix data structure is considerably smaller then the one related to the full matrix representation. Moreover, the memory consumption grows only linearly with the numbers of degrees of freedom instead of exponentially with the number of levels. This is the optimal result that one can expect, since a coefficient for each active basis function needs to be stored anyway.

We observe a difference between the results related to the rectangular-shaped refinement with respect to the linear and curvilinear case. The reason is the different nature of the refinement procedure. In the linear and curvilinear case, the refined area is reduced at each new level and the coarser levels do not change (see Figure 3). In the rectangular case, the refined area of the highest level is constant and the size of lower level subdomains increases (see Figure 2). Thus, using the sparse data structure does not decrease the order of memory consumption in this case, since the number of degrees of freedom grows exponentially with the number of levels.

The next example analyzes the influence of using the sparse data structures to the time needed to evaluate the multilevel spline functions using the algorithm EVAL_THB. 

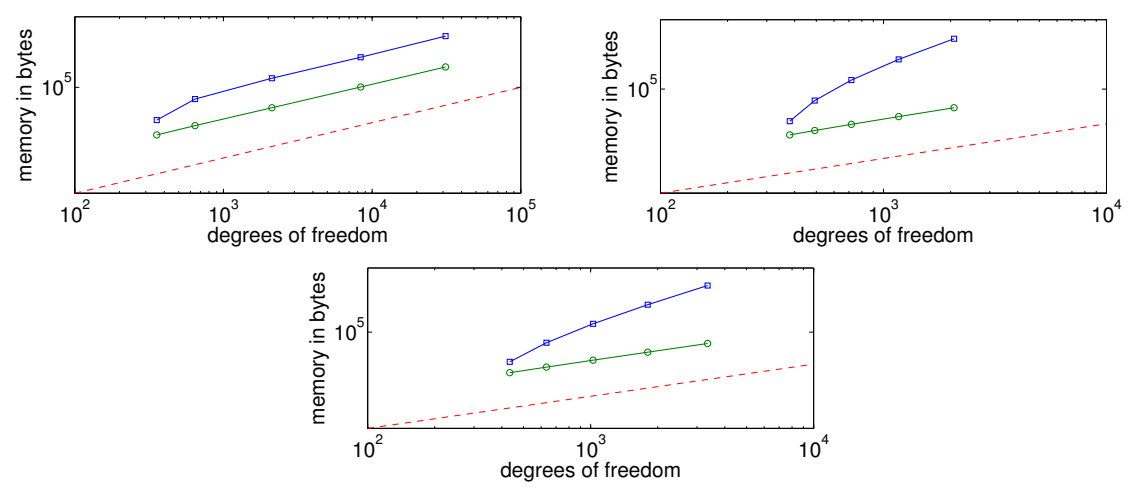

Fig. 10. Memory needed to represent the characteristic matrices without (blue) and with (green) the use of sparse data structures for different numbers of degrees of freedom related to the square (top left), the circle (top right) and the line refinement (bottom) refinement. The dashed red line has slope 1 and indicates linear growth.

Example 7. Figure 11 visualizes the distribution of the computation times needed to evaluate the multilevel spline function at 1000 points with (blue bars in the plot) and without (red bars in the plot) the use of sparse data structures for the linear refinement shown in Figure 9. Two facts can be observed:

- the evaluation time does not depend significantly on the location of the point with respect to the subdomain hierarchy;

- using the sparse data structure increases the evaluation time only by a very small amount.

Note that the evaluation times in this example vary between 0.153 and 0.195 milliseconds.

Finally we analyze the relation between evaluation time and the number of levels in the hierarchy.

Example 8. We consider the curvilinear refinement shown on the right of Figure 9. Figure 12 compares the evaluation times for 10,000 parameters obtained by using either the full or the sparse matrix representation. We may note that the computational time grows linearly with the increasing level of refinement for both representations, with a small overhead caused by using the sparse data structure. The values do not include the time necessary for creating the corresponding data structures, only the evaluation algorithm EVAL_THB is considered.

\section{Non-uniform knots and general refinement}

In order to discuss more general knot configurations, we now describe the modifications of data structures and algorithms which are required to extend the framework to non-uniform knots and different multiplicities. 


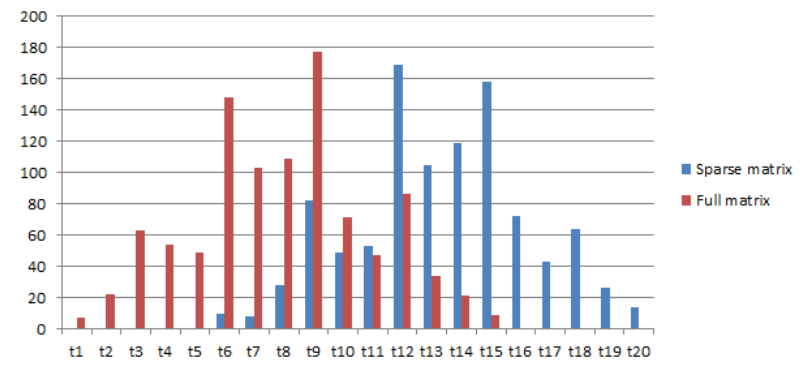

Fig. 11. The labels $t 1, \ldots, t 20$ on the horizontal axis represent uniform time intervals between minimal $(0.153 \mathrm{~ms})$ and maximal $(0.195 \mathrm{~ms})$ time needed by the evaluation algorithm. The vertical axis indicates the number of points whose evaluation time falls into these intervals.

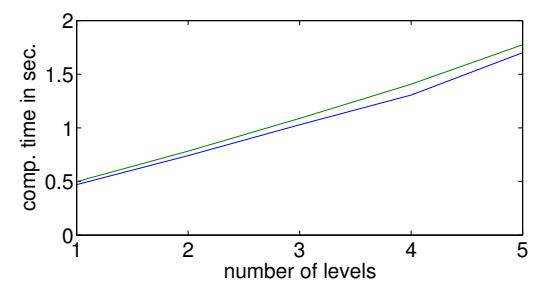

Fig. 12. Computational time needed to evaluate the multilevel spline function at 10,000 points for curvilinear refinement with various numbers of levels with (green) and without (blue) using the sparse data structure.

First of all, two vectors with strictly monotone knot values in both directions have to be stored. Two additional arrays will register the associated multiplicities. In this way, if we allow also knots with zero multiplicities, we can even perform non-dyadic refinements by simply considering to consecutive refinement levels where some of the newly inserted knots have multiplicity 0.

Concerning the modification of the THB-spline evaluation algorithm presented in Section 2, the only difference is in the use of B-spline subdivision with respect to non uniform knots, namely standard knot insertion, instead of uniform B-spline refinement. At each iteration, the knot insertion algorithm has to be applied to the proper sub-matrix of the corresponding matrix computed at the previous step.

In the quadtree data structure introduced in Section 3, the axis-aligned bounding box has now to be in index space and possibly of rectangular shape. In addition, the splitting into halves considered in the related insertion algorithm of Section 3.1 has to be rounded to the nearest integer. No modification are needed for the query functions described in Section 3.2. 
Finally, in order to let the characteristic matrices described in Section 4 properly identify the active B-spline functions, we just have to allow general rectangular structures and consider the index space of functions (instead of knots) into the two algorithms described therein.

\section{Conclusion}

We proposed an efficient implementation of data structures and related algorithms for the evaluation and manipulation of truncated hierarchical B-splines. Several examples show the advantageous behavior of the data structures and algorithms in terms of memory overheads and computational costs. Indeed, the memory consumption grows only linearly with the number of degrees of freedom, but there is no significant increase of the time needed to evaluate the multilevel spline function.

The generalization of the proposed algorithms to handle the non-uniform case and multiple knots can be addressed by considering the subdomain hierarchy in index space rather than in the physical one as described in Section 6. We are currently working on these more general configurations in the frame of a new software library which we are developing. Interesting subjects for future research include the extension to multivariate splines and the identification of the refinement algorithm for THB-splines.

Acknowledgments. Gábor Kiss is supported by the Austrian Science Fund (FWF) through the Doctoral Program in Computational Mathematics (W1214, DK3). Carlotta Giannelli is a Marie Curie Postdoctoral Fellow within the 7th European Community Framework Programme under grant agreement n ${ }^{\circ} 272089$ (PARADISE). Bert Jüttler has received support by the Austrian Science Fund (FWF) through the National Research Network Geometry + Simulation (S117). This research has also received funding from the Marie Curie Actions - IndustryAcademia Partnerships and Pathways (IAPP) funding scheme under grant agreement n³24340 (EXAMPLE).

\section{References}

1. Bornemann, P. B., and Cirak, F.: A subdivision-based implementation of the hierarchical B-spline finite element method, Comput. Methods Appl. Mech. Engrg., to appear (2012)

2. Deng, J., Chen, F. and Feng, Y.: Dimensions of spline spaces over T-meshes, J. Comput. Appl. Math. 194, 267-283 (2006)

3. Dokken, T., Lyche, T. and Pettersen, K. F.: Polynomial splines over locally refined box-partitions, Comput. Aided Geom. Design 30, 331-356 (2013)

4. Forsey, D. R., and Bartels, R. H.: Hierarchical B-spline refinement, Comput. Graphics 22, 205-212 (1988)

5. Giannelli, C., Jüttler, B., and Speleers, H.: THB-splines: the truncated basis for hierarchical splines. Comput. Aided Geom. Design 29, 485-498 (2012) 
6. Giannelli, C., Jüttler, B., and Speleers, H.: Strongly stable bases for adaptively refined multilevel spline spaces. Preprint (2012)

7. Giannelli, C., and Jüttler, B.: Bases and dimensions of bivariate hierarchical tensor-product splines, J. Comput. Appl. Math. 239,162-178 (2013)

8. Gilbert, J. R., Moler, C., and Schreiber, R.: Sparse matrices in MATLAB: design and implementation. SIAM J. Matrix Anal. Appl. 13, 333-356 (1992)

9. Gonzalez-Ochoa, C., and Peters, J., Localized-hierarchy surface splines (LeSS), In Proceedings of the 1999 symposium on Interactive 3D graphics, ACM, New York, NY, USA, 7-15 (1999)

10. Greiner G., and Hormann K.: Interpolating and approximating scattered 3D-Data with hierarchical tensor Product B-splines, In Méhauté, A. L., Rabut, C., Schumaker, L. L. (Eds.), Surface Fitting and Multiresolution Methods. In Innovations in Applied Mathematics. Vanderbilt University Press, Nashville, TN, 163-172 (1997)

11. Kraft, R.: Adaptive and linearly independent multilevel B-splines, in: Le Méhauté, A. and Rabut, C. and Schumaker, L. L. (Eds.), Surface Fitting and Multiresolution Methods, Vanderbilt University Press, Nashville, 209-218 (1997).

12. Kraft, R.: Adaptive und linear unabhängige Multilevel B-Splines und ihre Anwendungen, PhD Thesis, Universität Stuttgart (1998).

13. Lee, S., Wolberg, G., and Shin, S. Y.: Scattered data interpolation with multilevel B-splines, IEEE Trans. on Visualization and Computer Graphics 3, 228-244 (1997)

14. Schillinger, D., Dedè, L., Scott, M. A., Evans, J. A., Borden, M. J., Rank, E., and Hughes, T.J.R., An isogeometric design-through-analysis methodology based on adaptive hierarchical refinement of NURBS, immersed boundary methods, and T-spline CAD surfaces, Comput. Methods Appl. Mech. Engrg. 249-252, 116-150 (2012)

15. Schumaker, L. L. and Wang, L., Approximation power of polynomial splines on T-meshes, Comput. Aided Geom. Design 29, 599-612 (2012)

16. Sederberg, T. W., Zheng, J., Bakenov, A. and Nasri, A.: T-splines and TNURCCS, ACM Trans. Graphics 22, 477-484 (2003)

17. Stollnitz, E. J., DeRose, T. D., Salesin, D. H.: Wavelets For Computer Graphics: Theory and Application, first edition, Morgan Kaufmann Publishers, Inc. (1996)

18. Vuong, A.-V., Giannelli, C., Jüttler, B., and Simeon, B., A hierarchical approach to adaptive local refinement in isogeometric analysis, Comput. Methods Appl. Mech. Engrg. 200, 3554-3567 (2011)

19. Yvart A., and Hahmann S., Hierarchical triangular splines, ACM Trans. Graphics 24, 1374-1391 (2005) 